\title{
Cuestionario de sangrado preoperatorio en pacientes sometidos a amigdalectomía y/o adenoidectomía
}

\author{
Preoperative survey of bleeding in patients undergoing \\ tonsillectomy and or adenoidectomy
}

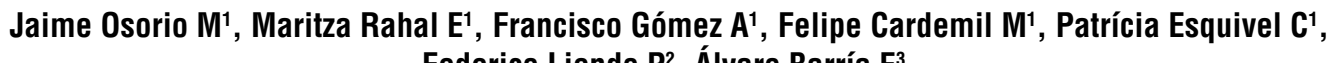
Federico Liendo P², Álvaro Barría $\mathrm{E}^{3}$.

\begin{abstract}
RESUMEN
Introducción: Se reporta entre 1\% y 6\% de hemorragia posamigdalectomía y/0 adenoidectomía. En la literatura se discute la real utilidad de los exámenes de hemostasia de rutina. Cuestionarios de sangrado estandarizados podrían definir a quién realizar un estudio de coagulación para predecir sangrados posamigdalectomía y/o adenoidectomía.

objetivo: Determinar la utilidad de un cuestionario preoperatorio para evaluar historia de sangrado y exámenes de coagulación rutinarios, para predecir sangrados asociados a amigdalectomía y/o adenoidectomía.

Material y método: Se realizó un estudio prospectivo de cohorte, en el Servicio de Otorrinolaringología, Hospital Barros Luco. Se incluyeron pacientes sometidos a amigdalectomía y/o adenoidectomía, con cuestionario a menores de 18 años, entre enero de 2008 y junio de 2010. Se usó Chi cuadrado, Fisher, $t$ de student según correspondiera para el análisis de los grupos. Se consideró estadísticamente significativo $p<0,05$.

Resultados: Se revisaron 951 fichas de pacientes operados en el período de estudio, de un total de 1.288 cirugías (73,8\%). Se excluyeron 65 por información incompleta y 272 sin cuestionario. El promedio (DE) de edad fue 7,70 $\pm 3,5$ años (Rango: 1 - 18 años). El $54 \%$ de los pacientes fue de sexo masculino. La frecuencia de sangrado fue $2,6 \%$. La razón de proporciones (OR) para edad fue 1,11 (IC 95\% 1,01-1,23); para amigdalitis crónica fue 2,56 (IC 95\% 1,15-5,69). Los exámenes presentaron una sensibilidad de 4\% y un valor de predicción positivo de $3 \%$. El cuestionario de sangrado preoperatorio presentó una sensibilidad de $24 \%$ y un valor de predicción positivo de $3 \%$.

Discusión: El bajo valor de predicción positivo del cuestionario y los exámenes (3\%) se asoció a la baja prevalencia de sangrado y otros factores involucrados en el sangrado posoperatorio. El cuestionario tuvo mayor capacidad de detectar sangrado posoperatorio (24\%). El uso del cuestionario representa una herramienta más confiable que los exámenes, pero menos costosa y menos traumática. Ante un cuestionario alterado se debe realizar estudio hematológico completo por especialista.

Conclusiones: El cuestionario preoperatorio es útil para discriminar a quién se le debe hacer estudio de coagulación. Los exámenes preoperatorios de rutina, el tiempo de protrombina (TP) y el tiempo de tromboplastina parcial activada (TTPa) en paciente sin antecedentes no parecen ser útiles.
\end{abstract}

\footnotetext{
Médico, Servicio de Otorrinolaringología, Hospital Barros Luco Trudeau, Universidad de Chile.

Médico del Servicio de Hematología, Hospital Barros Luco, Universidad de Chile.

Médico Cirujano, Universidad de Chile.
} 
Palabras clave: Cuestionario preoperatorio de sangrado, amigdalectomía y/o adenoidectomía, sangrado posoperatorio.

\begin{abstract}
Introduction: It is reported between $1 \%$ and $6 \%$ of post-tonsillectomy hemorrhage and / or adenoidectomy. The literature discusses the real usefulness of routine hemostasis tests. Standardized questionnaires bleeding could define who make a study of posttonsillectomy bleeding bleeding to predict and/or adenoidectomy.

Aim: To determine the usefulness of a preoperative questionnaire to assess history of bleeding and routine coagulation tests to predict bleeding associated with tonsillectomy and/or adenoidectomy.

Material and method: We performed a prospective cohort study in the Department of Otolaryngology, Hospital Barros Luco. We included patients undergoing tonsillectomy and / or adenoidectomy, with guest under 18 years between January 2008 and June 2010. We used chi-square, Fisher, Student $t$ test as appropriate for the analysis of the groups. Statistical significance was $p<0.05$.

Results: We reviewed 951 records of patients operated in the study period, a total of 1288 surgeries (73.8\%). 65 were excluded due to incomplete information and 272 without questionnaire. The mean (SD) age was $7.70+3.5$ years (range: 1 to 18 years). $54 \%$ of patients were male. The frequency of bleeding was $2.6 \%$. The ratio of ratios (OR) for age was 1.11 (95\% Cl 1.01 to 1.23) for chronic tonsillitis was 2.56 (95\% Cl 1.15 to 5.69). The tests showed a sensitivity of $4 \%$ and positive predictive value of $3 \%$. The preoperative bleeding questionnaire had a sensitivity of $24 \%$ and positive predictive value of $3 \%$.

Discussion: The low positive predictive value of the questionnaire and examinations (3\%) was associated with low prevalence of bleeding and other factors involved in postoperative bleeding. The questionnaire had a greater ability to detect postoperative bleeding (24\%). The use of the questionnaire represents a reliable tool that tests, but less expensive and less traumatic. Altered to a questionnaire study should be performed by complete blood specialist.

Conclusions: The preoperative questionnaire is useful to discriminate on who you should coagulation. The routine preoperative tests, prothrombin time (PT) and activated partial thromboplastin time (aPTT) in patients without a history not seem to help.

Key words: Questionnaire preoperative bleeding tonsillectomy and/or adenoidectomy, postoperative bleeding.
\end{abstract}

\section{INTRODUCCIÓN}

La adenoamigdalectomía es uno de los procedimientos quirúrgicos pediátricos más frecuentes'. Se reporta entre $1 \%$ y $6 \%$ de hemorragia posamigdalectomía y/o adenoidectomía 1 . Como causa de ésta se postulan varios factores, como la técnica quirúrgica, trauma operatorio y factores ambientales².

Las anormalidades congénitas 0 adquiridas de la hemostasia, como la enfermedad de von Willebrand, hemofilia, o uso de medicamentos, pueden predisponer a sangrado perioperatorio ${ }^{2}$.
La potencial presencia de un trastorno de la hemostasia, o razones médico-legales, ha llevado a algunos médicos a recomendar pruebas de coagulación preoperatoria en todos los pacientes que se someten a una amigdalectomía y/o adenoidectomía ${ }^{3}$. Sin embargo, la real utilidad de los exámenes de coagulación de rutina es cuestionada por la literatu$\mathrm{ra}^{4}$. Estudios sugieren que éstos deben ser hechos sólo en pacientes con historia o examen físico compatible con coagulopatía ${ }^{5}$.

La American Academy of Otolaryngology-Head and Neck Surgery aconseja la realización de exámenes sólo 
para aquellos pacientes cuyos antecedentes familiares o su historia médica sugiere un trastorno de la coagulación, o si la información genética sobre la familia biológica no es evaluable 6 . Esta postura también es adoptada por la Agencia Nacional de Acreditación y de Evaluación en Salud de Francia ${ }^{7}$, la Sociedad de Anestesiología de Chile ${ }^{8}$, la Sociedad Alemana de Otorrinolaringología ${ }^{9}$ y el Comité Británico de Normas en Hematología 5 . El nivel de evidencia de estas recomendaciones está basado en estudios bien formulados, pero no en ensayos clínicos aleatorios, lo cual genera controversia a la hora de aplicar estas recomendaciones ${ }^{5}$.

La aplicación de cuestionarios estandarizados de sangrado preoperatorio, facilitaría la identificación de los pacientes con potencial riesgo de sangrado posoperatorio y orientaría a cuáles pacientes se les debe realizar el estudio de coagulación ${ }^{1}$.

Como hipótesis se planteó que los cuestionarios de sangrado pueden definir a quién solicitar el estudio de coagulación para predecir sangrados posamigdalectomía y/o adenoidectomía.

Por ello el objetivo de este estudio fue determinar la utilidad del cuestionario preoperatorio para historia de sangrado y de los exámenes de coagulación de rutina para predecir hemorragias asociadas a amigdalectomía y/o adenoidectomía.

\section{OBJETIVO}

Como objetivos específicos se planteó determinar las características demográficas de los pacientes operados de amigdalectomía y/o adenoidectomía, la frecuencia de sangrado precoz (antes de las 24 horas) y tardío, los diagnósticos preoperatorios de coagulopatías y si requirió de algún procedimiento preoperatorio, analizar las diferencias entre los pacientes que presentaron hemorragia posoperatoria y los que no y, por último, el valor de predicción del cuestionario preoperatorio y de los exámenes de coagulación.

\section{MATERIAL Y MÉTODO}

Se realizó un estudio prospectivo de cohorte en pacientes usuarios del Hospital Barros Luco Trudeau (HBLT) operados de amigdalectomía y/o adenoidectomía, menores de 18 años, con cuestionario preoperatorio de sangrado respondido, y cuya cirugía hubiese sido efectuada entre enero de 2008 y junio de 2010. Se excluyeron los pacientes con datos incompletos y sin cuestionario.

El cuestionario (Anexo 1) se desarrolló en conjunto con el Departamento de Hematología del HBLT para es-

\section{Anexo 1. Cuestionario estandarizado preoperatorio de sangrado}

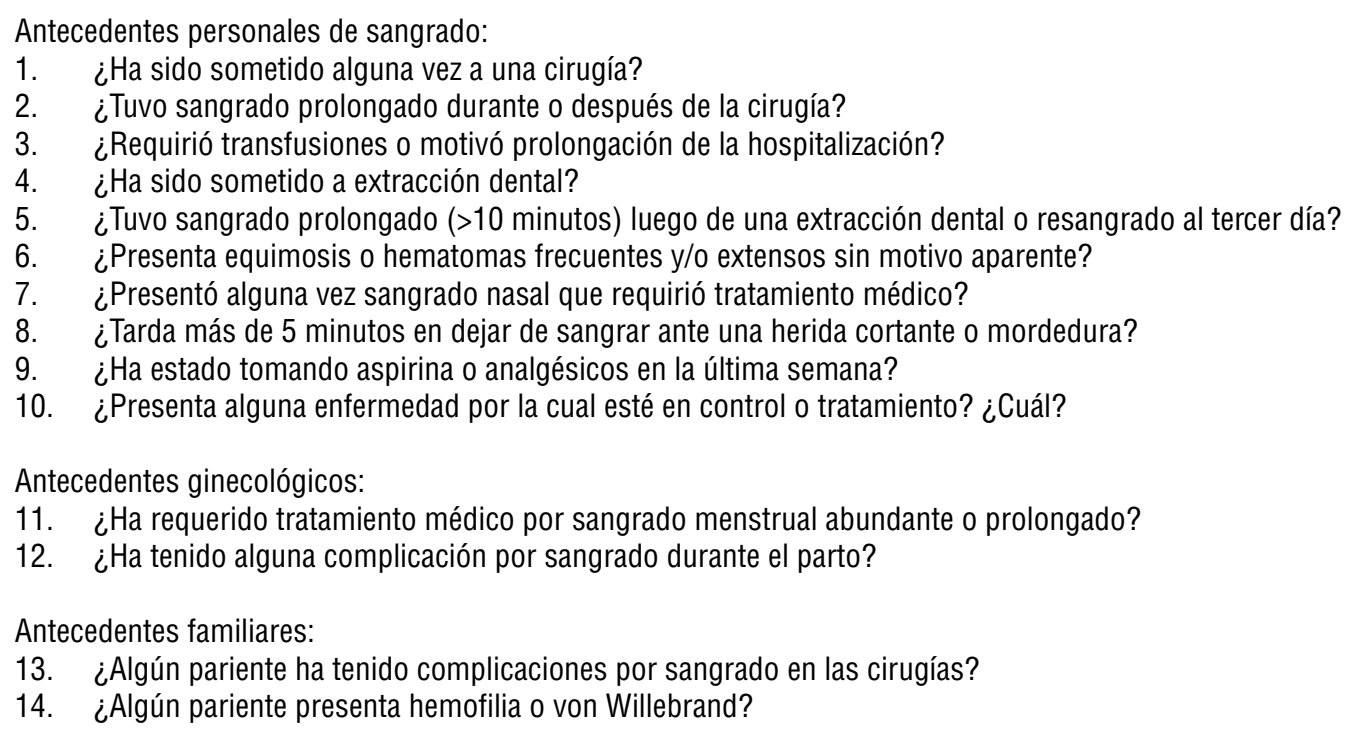


tandarizar la evaluación preoperatoria de los pacientes sometidos a amigdalectomía y/o adenoidectomía basado en la literatura internacional.

Se registró el diagnóstico por hematología y si se requirió manejo preoperatorio y operatorio para su intervención. Se consignó la edad, indicación de cirugía, cirugía realizada, técnica de resección quirúrgica, técnica de hemostasia, experiencia del cirujano, día de sangrado posoperatorio y manejo de sangrado posoperatorio.

Se consideró como encuesta anormal si presentaba una 0 más respuestas alteradas (siendo la primera y la cuarta preguntas introductoras). Se consideraron exámenes anormales cuando el tiempo de protrombina (TP) fue menor de $70 \%$, y/o el tiempo de tromboplastina parcial activada (TTPa) fue diez segundos mayor al valor de referencia, siendo en nuestro caso mayor de 36 segundos. A los pacientes con exámenes alterados se les repitieron éstos y, si persistían alterados, se les solicitó evaluación por hematólogo. Se consignó si presentaron diagnóstico por este especialista y si se realizó intervención perioperatoria.

Se definió sangrado intraoperatorio como una cirugía que demoró más de 2 horas debido a sangrado, o que éste fuera consignado por el cirujano en el protocolo quirúrgico.

Se definió sangrado posoperatorio en aquellos pacientes en que se constató sangrado después de la cirugía, teniendo que retornar a pabellón para controlar éste y/o que requirieron rehospitalización para su observación.

Para el análisis estadístico se utilizó el programa SPSS. Los resultados se expresaron como frecuencias absolutas y relativas para variables categóricas, y como promedios y desviaciones estándar (DE) las cuantitativas. Se utilizó prueba de Chi cuadrado, Fisher, y t de Student para el análisis de los grupos, según el tipo de variable. Se estimó un tamaño de muestra significativo de 753 pacientes, con alfa $5 \%, 80 \%$ de poder, y frecuencia de sangrado posoperatorio de $2 \%$. Se calculó sensibilidad, especificidad, y valores predictivos del cuestionario y exámenes de laboratorio. Se consideró estadísticamente significativo $p \leq 0,05$.

\section{RESULTADOS}

Se revisaron 951 de 1.288 fichas de cirugías, lo que correspondió al $73,8 \%$ de éstas. De las fichas no consideradas en este estudio, 65 (5\%) fichas presentaban información incompleta, y 272 $(21,1 \%)$ cirugías sin cuestionario respondido.

El promedio (DE) de edad fue de $7,7( \pm 3,5)$ años, con una edad mínima de 1 año y máxima de 18 años. El $54 \%$ fueron pacientes de sexo masculino.

La frecuencia de sangrado posoperatorio fue de $2,6 \%(n=25)$ para el grupo estudiado. De éstos, el $50 \%$ se presentó el primer día, y el resto alrededor del séptimo día posoperatorio (Figura 1). Se realizó manejo quirúrgico en el $72 \%$ de los casos de sangrado para control de la hemorragia, y sólo un paciente requirió transfusión de glóbulos rojos por anemia aguda.

De los pacientes sometidos a amigdalectomía y/o adenoidectomía, a 9 pacientes se les realizó el diagnóstico de coagulopatía (1\%) (Tabla 1). De éstos, todos presentaron cuestionario alterado, y 7 tuvieron exámenes de laboratorio anormales.

Ninguno de los pacientes con coagulopatía presentó sangrado posoperatorio. Los procedimientos

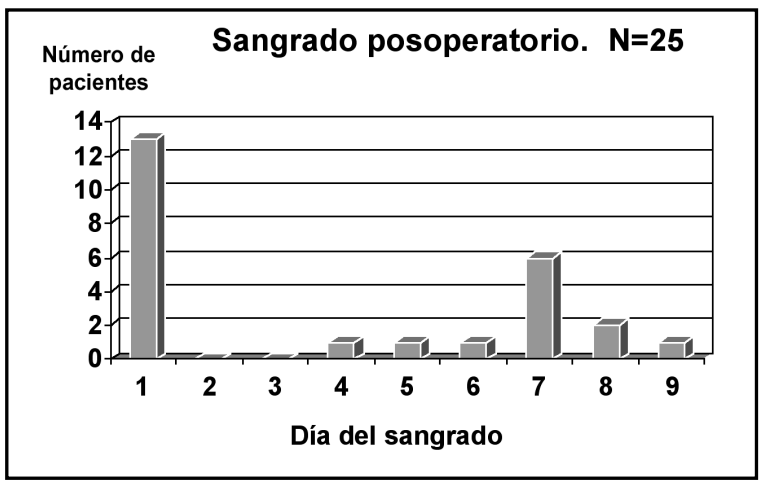

Figura 1. Distribución de pacientes con sangrado posoperatorio según día de sangrado. 
Tabla 1. Pacientes con diagnóstico hematológico sometidos a amigdalectomía y/o adenoidectomía

\begin{tabular}{|lrr|}
\hline Diagnóstico & $\mathrm{n}$ & $\%$ \\
\hline Coagulopatía por daño hepático & 3 & 33,3 \\
Hipoprotrombinemia & 2 & 22,2 \\
Déficit de factor VII & 2 & 22,2 \\
Hemofilia & 2 & 22,2 \\
Total & 9 & 100,0 \\
\hline
\end{tabular}

realizados antes de la cirugía se observan en la Tabla 2.

Al comparar los pacientes operados que presentaron sangrado se observa que los pacientes de mayor edad y aquellos con indicación quirúrgica por amigdalitis crónica tuvieron significativo mayor riesgo de sangrado, con un OR de 1,1 para edad, y 2,56 para amigdalitis crónica. En cambio, la cirugía realizada, sexo, técnica de resección quirúrgica, técnica de hemostasia, años de experiencia del cirujano, exámenes de coagulación alterados, e historia de hemostasia alterada, no se asoció a mayor riesgo de sangrado (Tabla 3).

Se encontró relación entre un cuestionario alterado y la probabilidad de tener un examen alterado; esto es, los pacientes con antecedentes personales o familiares de sangrado tuvieron más probalidades de presentar de exámenes alterados (Tabla 4).

No existió relación significativa entre la historia de sangrado y el sangrado posoperatorio, así como con los resultados de exámenes de coagulación de rutina. Las Tablas 5 y 6 muestran que gran parte de los pacientes que presentan hemorragia tienen historia y exámenes normales.
Respecto a la validez, se observó que el cuestionario presenta mayor sensibilidad para diagnosticar un sangrado posoperatorio (24\%) que los exámenes realizados en el preoperatorio (4\%) (Tabla 7).

Tanto los exámenes como el cuestionario preoperatorio tuvieron un valor de predicción positivo muy bajo para prever un sangrado posoperatorio (3\%); por ende, son muy inseguros en este aspecto (Tabla 7).

\section{DISCUSIÓN}

La amigdalectomía y/o adenoidectomía se asocia a sangrado posoperatorio y otras complicaciones menos frecuentes. Muy rara vez se ha descrito mortalidad (1:35.000-50.000) $)^{10-18}$.

La frecuencia de sangrado y las características demográficas encontradas en este estudio concuerdan con la literatura internacional. La edad y la amigdalitis crónica se asociaron a mayor riesgo de sangrado, al igual que lo señalado en la literatura ${ }^{10-18}$.

El porcentaje de pacientes con coagulopatía diagnosticada previo a la cirugía fue de $1 \%$, en contraste con el $4 \%$ de exámenes anormales y el $23 \%$ de cuestionarios alterados.

Tabla 2. Procedimientos preoperatorios en pacientes sometidos a amigdalectomía y/o adenoidectomía

\begin{tabular}{|lcr|}
\hline Procedimiento & $\mathrm{n}$ & $\%$ \\
\hline Vitamina K & 4 & 44,4 \\
Espercil & 3 & 33,3 \\
Plasma fresco congelado & 1 & 11,1 \\
Glóbulos rojos por anemia & 1 & 11,1 \\
Total & 9 & 100,0 \\
\hline
\end{tabular}


Tabla 3. Sangrado posoperatorio de pacientes sometidos a amigdalectomía y/o adenoidectomía según variables

\begin{tabular}{|c|c|c|c|c|}
\hline Variable & & $\begin{array}{l}\text { No Sangrado } \\
n=926\end{array}$ & $\begin{array}{c}\text { Sangrado } \\
\mathrm{n}=25\end{array}$ & Valor de $p$ \\
\hline Sexo & Masculino & $500(54 \%)$ & $12(48 \%)$ & $0,685^{\star}$ \\
\hline Edad (DE) & & $7,65 \pm 3,4$ & $9,16 \pm 4,5$ & $0,03^{* * *}$ \\
\hline \multirow[t]{3}{*}{ Indicación quirúrgica } & Amigdalitis & & & \\
\hline & $\begin{array}{l}\text { crónica } \\
\text { Hiperplasia }\end{array}$ & $245(27 \%)$ & $12(48 \%)$ & $0,022^{*}$ \\
\hline & adenoamigdalina & $681(73 \%)$ & $13(52 \%)$ & \\
\hline \multirow{2}{*}{ Cirujano } & Staff & $553(60 \%)$ & $17(68 \%)$ & $0,536^{\star}$ \\
\hline & Residente & $373(40 \%)$ & $8(32 \%)$ & \\
\hline \multirow[t]{3}{*}{ Cirugía } & Amigdalectomía & $383(41 \%)$ & $10(40 \%)$ & $0,989^{* *}$ \\
\hline & $\begin{array}{l}\text { Amigdalectomía con } \\
\text { adenoidectomía }\end{array}$ & $468(51 \%)$ & $13(52 \%)$ & \\
\hline & Adenoidectomía & $75(8 \%)$ & $2(8 \%)$ & \\
\hline \multicolumn{5}{|l|}{ Método de resección } \\
\hline & Disección fría & $883(95 \%)$ & $24(96 \%)$ & $0,706^{\star \star}$ \\
\hline & Electrodisección & $24(3 \%)$ & $1(4 \%)$ & \\
\hline & Radiofrecuencia & $19(2 \%)$ & 0 & \\
\hline \multicolumn{5}{|l|}{ Método de hemostasia } \\
\hline & Clásica & $496(54 \%)$ & $11(44 \%)$ & $0,562^{* *}$ \\
\hline & Electrocoagulación & $65(7 \%)$ & $1(4 \%)$ & \\
\hline & Radiofrecuencia & $64(7 \%)$ & $3(12 \%)$ & \\
\hline & Clásica + electro & $301(32 \%)$ & $10(40 \%)$ & \\
\hline $\begin{array}{l}\text { Ex. coagulación } \\
\text { alterados }\end{array}$ & & $34(4 \%)$ & $1(4 \%)$ & $0.931^{*}$ \\
\hline Cuestionario anormal & & $212(23 \%)$ & $6(24 \%)$ & $0,812^{*}$ \\
\hline
\end{tabular}

${ }^{\star}$ Fisher; ${ }^{\star \star}{ }^{*} \mathrm{Chi}^{2} ;{ }^{* \star \star}$ t de Student

OR para edad: $1,11(1,01-1,23) p<0,038$.

OR para amigdalitis crónica: $2,56(1,15-5,69) p<0,021$.

DE: Desviación estándar

Tabla 4. Relación entre la historia de sangrado y exámenes de laboratorio

\begin{tabular}{|lcc|}
\hline Historia de sangrado & \multicolumn{2}{c|}{ Exámenes de laboratorio } \\
& $\begin{array}{c}\text { Alterados } \\
(\mathrm{n}=35)\end{array}$ & $\begin{array}{c}\text { No alterados } \\
(\mathrm{n}=916)\end{array}$ \\
\hline Positiva & $16(45,7 \%)$ & $202(22,1 \%)$ \\
Negativa & $19(54,3 \%)$ & $714(77,9 \%)$ \\
\hline
\end{tabular}

Fisher p: 0,03

Ninguno de los pacientes con coagulopatía presentó sangrado posamigdalectomia, lo cual probablemente se explique por la preparación previa ${ }^{19-22}$.
El uso de TP y TTPa para el diagnóstico de coagulopatias es insuficiente, ya que no hay concordancia entre éstos, las historias de hemorragias, 
Tabla 5. Asociación de sangrado posoperatorio con cuestionario

\begin{tabular}{|lcc|}
\hline Historia de sangrado & \multicolumn{2}{c|}{ Sangrado posoperatorio } \\
& $\begin{array}{c}\text { Pacientes con sangrado } \\
\text { posoperatorio } \\
(\mathrm{n}=25)\end{array}$ & $\begin{array}{c}\text { Pacientes sin sangrado } \\
\text { posoperatorio } \\
(\mathrm{n}=926)\end{array}$ \\
\hline Positivo & $06(24 \%)$ & $212(22,9 \%)$ \\
Negativo & $19(76 \%)$ & $714(77,1 \%)$ \\
\hline
\end{tabular}

Fisher: NS

Tabla 6. Asociación de sangrado posoperatorio con exámenes

\begin{tabular}{|c|c|c|}
\hline \multirow[b]{2}{*}{ Exámenes de laboratorio } & \multicolumn{2}{|c|}{ Sangrado posoperatorio } \\
\hline & $\begin{array}{l}\text { Pacientes con sangrado } \\
\text { posoperatorio } \\
(n=25)\end{array}$ & $\begin{array}{l}\text { Pacientes sin sangrado } \\
\text { posoperatorio } \\
(n=926)\end{array}$ \\
\hline Positiva & $01(4 \%)$ & $34(3,7 \%)$ \\
\hline Negativa & $24(96 \%)$ & $892(96,3 \%)$ \\
\hline
\end{tabular}

Fisher: NS

Tabla 7. Evaluación de las pruebas diagnósticas para sangrado posoperatorio

\begin{tabular}{|lcccc|}
\hline & Sensibilidad & Especificidad & Valor predictivo $(+)$ & Valor predictivo $(-)$ \\
\hline Ex. coagulación & 0,04 & 0,96 & 0,03 & 0,97 \\
Cuestionario preoperatorio & 0,24 & 0,77 & 0,03 & 0,97 \\
\hline
\end{tabular}

y el sangrado posoperatorio. Por ejemplo, muchos de los pacientes con enfermedad de von Willebrand sintomática, tienen TP y TTPa normales ${ }^{19-22}$. Para el adecuado diagnóstico de coagulopatías no sólo se debe realizar TP y TTPa, sino que se requiere de exámenes más específicos y costosos ${ }^{19-22}$.

El bajo valor de predicción positivo del cuestionario y los exámenes (3\%) se puede explicar por la baja prevalencia de sangrado y a otras causas de sangrado posoperatorio. El cuestionario tuvo mayor capacidad de detectar sangrado posoperatorio pero, con un bajo porcentaje (24\%). La mayoría de los pacientes que presentaron sangrado posoperatorio tuvieron exámenes y cuestionario normal, por lo que es necesario buscar otros factores que expliquen el sangrado, no existiendo en la actualidad buenos elementos de predicción de su presencia en el posoperatorio ${ }^{23-30}$.

Existe controversia en la literatura respecto a realizar exámenes preoperatorios llamados "de rutina" en pacientes sin antecedentes clínicos que hagan sospechar una alteración de la coagulación. Se esgrimen razones como experiencia personal, problemas médico-legales, política del hospital 0 disponer de recursos ilimitados para la solicitud de exámenes de rutina². 
En la Tabla 8 se observan las recomendaciones de los diferentes estudios publicados en la literatura anglosajona desde el año 1970 a la fecha. Estos trabajos son estudios clínicos bien diseñados, algunos retrospectivos y otros prospectivos, lo cual representa un grado de evidencia tipo III y nivel de recomendación $\mathrm{B}^{5,10,21}$.

Gabrie $^{23}$ y Burk ${ }^{27}$ efectuaron estudios prospectivos con muestras significativas, en donde el valor de predicción positivo para los exámenes de coagulación de rutina fue similar a lo encontrado en nuestro estudio. Ellos recomendaron realizar un estudio selectivo de los pacientes sometidos a adenoamigdalectomía. Nuestro trabajo, al igual que la literatura internacional, reafirma el concepto de que el estudio de coagulación debe ser dirigido a pacientes con antecedentes de coagulopatía y no solicitarlo universalmente, lo que se puede extrapolar a otras patologías con menor incidencia de sangrado.

El uso del cuestionario representaría una herramienta igualmente confiable que los exámenes, pero menos costosa y menos traumática. El uso de la encuesta ahorraría aproximadamente $\$ 7.320$ - por cada procedimiento quirúrgico amigdalino. En Chile durante el año 2004, se realizaron 20.000 amigdalectomías, por lo que anualmente en nuestro país se ahorraría un total cercano a los $\$ 146.400 .000$, sólo por concepto de estos exámenes ${ }^{4,10,31}$.
Si bien el presente estudio no es aleatorio, ya que éticamente no es posible realizarlo, éste nos permite tomar exámenes a la población de riesgo y realizar estudio de coagulación a pacientes con historia de sangrado, al igual que lo relatado en experiencias internacionales ${ }^{1}$. Este estudio presenta el sesgo que no todos los pacientes fueron evaluados por hematología para conocer la real incidencia de trastorno de la hemotasia de nuestra muestra; para ello se debe estudiar en forma completa los pacientes con historia de sangrado mediante estudio de factores de coagulación como VIII, IX, VII, factor de von Willebrand, agregación plaquetaria y hemograma completo.

Se ha planteado las implicancias médico-legales de no tomar exámenes de coagulación de rutina, en base a estudios 0 temores en la práctica habitual, lo cual debe ser confrontado por la evidencia internacional y nacional. Esto debiera apoyar la realización de consensos nacionales tendientes a adoptar políticas de salud pública acorde con estos lineamientos.

\section{CONCLUSIONES}

El cuestionario preoperatorio para historia de sangrado y los exámenes de coagulación de rutina son malos para predecir sangrado posoperatorio,

Tabla 8. Recomendación de realizar exámenes de coagulación de rutina en pacientes adenoamigdalectomizados

\begin{tabular}{|lrrrrc|}
\hline Estudio & n Total & \multicolumn{2}{c}{ Exámenes } & VPP & Recomendación \\
& \multicolumn{7}{c}{$\begin{array}{c}\text { alterados } \\
\text { del estudio }\end{array}$} \\
\hline Shaw et al. 2008 & 842 & 23 & 2,7 & - & Selectivo \\
Eisert et al. 2006 & 148 & 27 & 18,24 & - & Selectivo \\
Asaf et al. 2001 & 416 & 237 & 56,8 & 0,09 & Selectivo \\
Gabriel et al. 2000 & 1.463 & 57 & 3,9 & 0,16 & Selectivo \\
Zwack and Derkay 1997 & 4.370 & - & - & - & Selectivo \\
Howells et al. 1997 & 339 & 39 & 11,5 & 0,03 & Selectivo \\
Kang et al. 1994 & 1.069 & 27 & 2,5 & 0,22 & Rutina \\
Close et al. 1994 & 96 & 20 & 20,8 & 0 & Selectivo \\
Burk et al. 1992 & 1.603 & 31 & 1,93 & 0,06 & Selectivo \\
Bolger et al. 1990 & 52 & 14 & 26,9 & - & Rutina \\
Smith et al. 1990 & 250 & 44 & 17,6 & - & Rutina \\
Manning et al. 1987 & 994 & 58 & 5,8 & 0,03 & Selectivo \\
Tami et al. 1987 & 775 & 74 & 9,6 & - & Rutina \\
Handler et al. 1986 & 1.445 & - & - & - & Rutina \\
Thomas and Arbon 1970 & 206 & 0 & 0 & - & Rutina \\
\hline
\end{tabular}


pero el uso del cuestionario preoperatorio es útil para discriminar a quién se le debe hacer estudio de coagulación. Los exámenes preoperatorios de rutina TP y TTPa en pacientes sin antecedentes sugieren no ser útiles.

El cuestionario preoperatorio presenta una mayor sensibilidad que los exámenes TP y TTPa para identificar a los pacientes con mayor riesgo de sangrado.

El cuestionario preoperatorio de sangrado es más eficaz y rentable que los exámenes de rutina.

Contando con el respaldo de la evidencia nacional e internacional, se sugiere realizar sólo el cuestionario como método de selección, y solicitar un estudio hematológico si fuese pertinente.

\section{BIBLIOGRAFÍA}

1. Licameli $G$, Jones $D$, Santosuosso J. Use of a preoperative bleeding questionnaire in pediatric patients who undergo adenotonsillectomy. Otolaryngol Head Neck Surg 2008; 139: 546-50.

2. Wieland A, Belden L, Cunningham M. Preoperative coagulation screening for adenotonsillectomy: A review and comparison of current physician practices. Otolaryngol Head Neck Surg 2009; 140: 542-7.

3. Derkay C. Cost-effective approach for preoperative hemostatic assessment in children undergoing adenotonsillectomy. Arch Otolaryngol Head Neck Surg 2000; 126: 68.

4. Namoncura C, Breinbauer H. Utilidad y costoefectividad de los exámenes preoperatorios en cirugía otorrinolaringológica. Rev Otorrinolaringol Cir Cabeza Cuello 2009; 69: 117-24.

5. Chee y, Crawford J, Watson H y col. Guidelines on the assessment of bleeding risk prior to surgery or invasive procedures. British Committee for Standards in Haematology. Br J Haematol 2008; 140: 496-504.

6. The Academy of Otolaryngology-Head and Neck Surgery. Clinical Indicators Compendium. Alexandria, VA: American Academy of Otolaryngology-Head and Neck Surgery; 1999.

7. Agence Nationale d'Accreditation et d'Évaluation en Santé, Service des recommandations et références professionnelles. Les examens préopératoires systématiques. Paris, 1998.
8. Sociedad de Anestesiología de Chile.

9. Recomendaciones en la evaluación preoperatoria pediátrica. Disponible en: http://www.sachile.cl/ medicos/recomendaciones/pre_anestesica/ evaluacion_nino.php [Consultado el 01 de Enero de 2011].

10. Scheckenbach K, Bier H, Hoffmann TK et al. Risk of hemorrhage after adenoidectomy and tonsillectomy. Value of the preoperative determination of partial thromboplastin time, prothrombin time and platelet count. HNO2008; 56: 312-20.

11. Cooper J, Smith K, Ritchey A. Cost-effectiveness analysis of coagulation testing prior to tonsillectomy and adenoidectomy in children. Pediatr Blood Cancer 2010; 55: 1045-6.

12. Gallagher T, Wilcox L, McGuire E, Derkay C. Analyzing factors associated with major complications after adenotonsillectomy in $\mathbf{4 7 7 6}$ patients: Comparing three tonsillectomy techniques. Otolaryngol Head and Neck Surg 2010; 142: 886-92.

13. BLAKLEY B. Post-tonsillectomy bleeding: How much is too much? Otolaryngol Head Neck Surg 2009; 140: 288-90.

14. BlaKLey B, Magit A. The role of tonsillectomy in reducing recurrent pharyngitis: A systematic review. Otolaryngol Head Neck Surg 2009; 140: 291-7.

15. Schrock A, Send T, Heukamp L, et al. The role of histology and other risk factors for posttonsillectomy haemorrhage. Eur Arch Otorhinolaryngol 2009; 266: 1983-7.

16. Prima M, Diegoa J, Jimenez-Yusteb V, et al. Analysis of the causes of immediate unanticipated bleeding after pediatric adenotonsillectomy. Int J Pediatr Otorhinolaryngol 2003; 67: 341-4.

17. Krishna P, Lee D. Post-tonsillectomy bleeding: A meta-analysis. Laryngoscope 2001; 111(8): 1358-61.

18. Randall DA, Hoffer ME. Complications of tonsillectomy and adenoidectomy. Otolaryngol Head Neck Surg 1998; 118(1): 61-8.

19. Myssiorek D, Alvi A. Post-tonsillectomy hemorrhage: an assessment of risk factors. Int J Pediatr Otorhinolaryngol 1996; 37: 35-43.

20. MarionI G, FILIPPIS C. Pediatric otolaryngologic manifestations of bleeding disorders. Int Pediatr Otorhinolaryngol 2009; 73S: S61-S64. 
21. Verdugo P, Morales M, Quiroga T. Correlación clínica y de laboratorio en pacientes pediátricos portadores de enfermedad de von Willebrand. Rev Ped Elec 2005; 2 (2): 5-10.

22. Windfunt J, Chen YS, Remmert S. Unidentified coagulation disorders in post-tonsillectomy hemorrhage. Ear Nose Throat J 2004; 83: 2839.

23. Sramek A, Eikenboom JC, Briet E, et al. Usefulness of patient interview in bleeding disorders. Arch Intern Med 1995; 155: 1409-15.

24. Gabriel P, Mazoit X, Ecoffey C. Relationship between clinical history, coagulation tests, and perioperative bleeding during tonsillectomies in pediatrics. J Clinical Anesth 2000; 12: 288-91.

25. Zwack G, Derkay C. The utility of preoperative hemostatic assessment in adenotonsillectomy. Int J Pediatr Otorhinolaryngol 1997; 39: 67-76.

26. Asaf T, Reuveni H, Yermiahu T, et al. The need for routine preoperative coagulation screening tests (prothrombin time PT/ partial thromboplastin time PTT) for healthy children undergoing elective tonsillectomy and/or adenoidectomy. Int J Pediatr Otorhinolaryngol 2001; 61: 217-22.
27. Houry S, Georgeac C, Hay JM, et al. A prospective multicenter evaluation of preoperative hemostatic screening tests. The French Associations for Surgical Research. Am J Surg 1995; 170: 19-23.

28. Burk CD, Miller L, Handler S, et al. Preoperative history and coagulation screening in children undergoing tonsillectomy. Pediatrics 1992; 89: 691-5.

29. Rohrer M, Michelotti M, Nahrwold D. Prospective evaluation of the efficacy of preoperative coagulation testing. Ann Surg 1988; 208 (5): 554-7.

30. Howells RC, Wax MK \& Ramadan HH. Value of preoperative prothrombin time/partial thromboplastin time as a predictor of postoperative hemorrhage in pediatric patients undergoing tonsillectomy. Otolaryngol Head Neck Surg 1997; 117: 628-2.

31. ZAGóLSKI 0. Hemorragia postamigdalectomía: ¿Tienen las pruebas de coagulación y el historial de coagulopatía un valor predictivo? Acta Otorrinolaringol Esp 2010; 61(4): 287-92.

32. Egresos hospitalarios 2004. Departamento de estadística e información de salud. MINSAL.

Dirección: Dr. Jaime Osorio M.

Servicio de Otorrinolaringología, Hospital Barros Luco Trudeau

Universidad de Chile

E mail: secretaria@sochiorl.cl 